\title{
Paediatric rheumatology: a subspecialty in its infancy that is making leaps and bounds
}

Pei Ling $\underline{\mathrm{Ooi}}^{1}$, MBBS, MRCPCH, Lynette Pei-Chi $\underline{\text { Shek }}^{1,2}$, MBBS, MRCP

ABSTRACT Paediatric rheumatology is an exciting field exploding with new knowledge of autoimmune and autoinflammatory conditions and how to treat them. It is, however, a relatively new subspecialisation in Asia. There is thus a great need to educate the public and medical community about paediatric rheumatic diseases so that children with such conditions are given the highest possibility of achieving normal function in their daily lives.

Keywords: juvenile arthritis, paediatric rheumatology

Five-year-old Little I tottered into the consultation room with her mother. The child was referred for further evaluation, as her mother was concerned about Little J's recent difficulty in moving her fingers. Little J started walking late and had "funny joints" for years. Having seen many doctors, she was finally diagnosed with arthrogryposis (congenital joint contractures). Her mother was taught physiotherapy to help stretch Little J's joints, but minimal improvement was seen. On examination of Little J, it became clear that the reason for the decreased range of movement of her joints was arthritis.

Childhood-onset rheumatic diseases consist of many fascinating diseases specific to children and adolescents that are rarely encountered by the generalist. Detecting abnormalities in the musculoskeletal system of children is often challenging because of the presence of thicker subcutaneous fat and the variability of the anatomy of joints and bones at different stages of their growth. Therefore, the diseases often present as diagnostic dilemmas, and delays in therapy can potentially lead to significant morbidity.

Although chronic childhood arthritis was first described more than one hundred years ago, paediatric rheumatology was only recognised as a subspecialty less than 50 years ago. Since then, there has been increasing recognition by the international medical community concerning the need to care for and improve the outlook of affected children. The role of a paediatric rheumatologist is crucial for dealing with paediatric rheumatologic conditions not seen in adults (e.g. juvenile idiopathic arthritis [JIA], juvenile dermatomyositis, periodic fever syndromes, neonatal lupus, Kawasaki disease, Henoch-Schönlein purpura) and managing multiple paediatric issues for which physicians caring for adult patients tend to have limited understanding. These include growth and puberty, evaluation of pain in children (especially those who are nonverbal), use of antirheumatic drugs in children, the art of dealing with parents, school problems and transition into adulthood. ${ }^{(1)}$
Paediatric rheumatologists work with a paediatrician or family physician to evaluate and treat conditions involving the musculoskeletal system, and autoimmune or autoinflammatory disorders. A referral to a paediatric rheumatologist should be made for evaluation of:

- Arthritis or enthesitis - swelling, tenderness, warmth, erythema and/or limitation of movement of joints or tendon/ ligament insertions (entheses).

- Autoimmune disorders - systemic lupus erythematosus, scleroderma, vasculitis, inflammatory disorders of the muscle such as dermatomyositis or inflammatory disorders of the eyes/other organs.

- Evaluation of prolonged or recurrent fever.

- Unexplained complaints of chronic musculoskeletal pain, weakness, poor appetite or fatigue.

- Unexplained symptoms such as rash, weight loss, anaemia or joint swelling.

In Singapore, a formal paediatric rheumatology service was set up in National University Hospital (NUH) in 2002 and KK Women's and Children's Hospital (KKH) in 2009.(2) Prior to that, children with rheumatologic diseases were seen by rheumatologists, dermatologists and paediatricians at KKH, Tan Tock Seng Hospital, National Skin Centre and Singapore General Hospital. ${ }^{(3)}$ In 1998, the prevalence of childhood-onset rheumatologic diseases was only 178 cases over a three-year period, (3) but by 2012, the number had increased by approximately nine times. ${ }^{(2)}$ This rise in numbers likely reflects an increasing awareness of childhood-onset rheumatic diseases in Singapore, rather than a true increase in disease prevalence.

In Singapore, we are fortunate to have access to many of the newest advances in medical therapy for paediatric rheumatologic conditions. In addition to classic diseasemodifying antirheumatic drugs (DMARDs) such as methotrexate and sulphasalazine, many biologics with targeted therapy are also available for use in conditions such as JIA and paediatric

\footnotetext{
${ }^{1}$ Department of Paediatrics, Khoo Teck Puat-National University Children's Medical Institute, National University Hospital, ${ }^{2}$ Department of Paediatrics, Nationa University of Singapore, Singapore

Correspondence: Dr Ooi Pei Ling, Registrar, Department of Paediatrics, Level 12, NUHS Tower Block, 1E Kent Ridge Road, Singapore 119228,
} pei_ling_ooi@nuhs.edu.sg 
lupus. These biological agents include anti-tumour necrosis factor (TNF), anti-interleukin-1 and -6, anti-CD20, and more recently, anti-B-lymphocyte stimulator. Intra-articular steroid injections for arthritis in children have also been shown to be a safe and effective therapeutic option. This is supported by an audit on intra-articular steroid injections performed in JIA patients in NUH. ${ }^{(4)}$ The audit showed that joint injections were performed only once in $80.8 \%$ of patients with good clinical response and that no incidence of septic arthritis or avascular necrosis was recorded. Physiotherapy and occupational therapy complement therapeutic effort to achieve complete disease quiescence, enabling the child to have a normal life and function.

Paediatric rheumatology is clearly an increasingly recognised subspecialty. It is exciting to have so many more options to treat paediatric rheumatic diseases today compared to 20 years ago. We no longer have to accept that a child with a paediatric rheumatic condition will appear cushingoid or become disabled. Moving forward, we need to educate the public and medical community about paediatric rheumatic diseases so that children with such conditions can receive early and directed therapy so as to minimise morbidity.

Little I was diagnosed with polyarticular JIA and treated with an anti-TNF biologic. Within two months, she could straighten her knee, ankle and elbow joints, and was noticeably more cheerful. Her mother and schoolteachers were happy to see such a big change in her. Her paediatric rheumatologist was even happier to know that this child no longer needs to suffer from this painful disease.

\section{REFERENCES}

1. Spencer $\mathrm{CH}$. Why should pediatric rheumatology be recognized as a separate subspecialty: an open letter to medical councils and government agencies. Pediatr Rheumatol Online J 2007; 5:21.

2. JH Tan, Fun HS, Arkachaisri T. Paediatrics rheumatology clinic population in Singapore: The KKH experience. Proc Singapore Healthc 2012; 21:265-71.

3. See Y, Koh ET, Boey ML. One hundred and seventy cases of childhoodonset rheumatological disease in Singapore. Ann Acad Med Singapore 1998; 27:496-502.

4. Leow OM, Lim LK, Ooi PL, et al. Intra-articular glucocorticoid injections in patients with juvenile idiopathic arthritis in a Singapore hospital. Singapore Med J 2014; 5:248-52. 